\title{
EXPRESSION OF HMGB1 AND HMGN2 IN GINGIVAL TISSUES, GCF AND PICF OF PERIODONTITIS PATIENTS AND PERI-IMPLANTITIS
}

\author{
Ping Xie ${ }^{1}$, Lu-Xia Deng ${ }^{2,3}$, Ping Gong ${ }^{1}$, Yi Ding ${ }^{1}$, Xiao-Hai Tang ${ }^{2,3,4^{*}}$ \\ ${ }^{1}$ State Key Laboratory of oral Diseases, West China College of Stomatology, Sichuan University, Chengdu, China; ${ }^{2}$ Chongqing \\ Lummy Pharmaceutical Co., Ltd. Chongqing, China; ${ }^{3}$ State Key Laboratory of Biotherapy, West China Hospital, West China \\ Medical School, Sichuan University, Chengdu, China; ${ }^{4}$ Department of Gynecological Oncology, Sichuan Cancer Hospital,
} Chengdu, China.

Submitted: August 18, 2010; Approved: May 16, 2011.

\begin{abstract}
High mobility group chromosomal protein B1 (HMGB1) and N2 (HMGN2), two members of High mobility group (HMG) family, play important role in inflammation. The purposes of this study were to investigate the expression of HMGB1 and HMGN2 in periodontistis.

The expression of HMGB1 and HMGN2 mRNA in gingival tissues and gingival crevicular fluid (GCF) in chronic periodontitis $(\mathrm{CP})$, generalized aggressive periodontitis (G-AgP) patients and healthy subjects was detected by real-time PCR. The protein level of HMGB1 and HMGN2 in peri-implant crevicular fluid (PICF), peri-implant crevicular fluid of peri-implantitis (PI-PICF) and normal patients was determined by Western blotting. Furthermore, IL-1 $\beta$, IL-6, IL-8, TNF- $\alpha$ and HMGB1 levels in GCF, PI-PICF and healthyPICF samples from different groups were determined by ELISA.

HMGN2 expression was increased in inflamed gingival tissues and GCF from CP and G-ApG groups compared to control group. HMGB1 expression was the highest in the gingival tissues and GCF from CP patients and was accompanied by increased concentrations of IL-1 $\beta$, IL-6, IL-8 proinflammaory cytokines. To our knowledge, this is the first study reporting that the expression of HMGB1 and HMGN2 was increased in the gingival tissues and GCF in $\mathrm{CP}$ and G-AgP and the PICF in PICF. Our data suggest that HMGB1 may be a potential target for the therapy of periodontitis and PI.
\end{abstract}

Key words: HMGB1; HMGN2; periodontitis; peri-implantitis

\section{INTRODUCTION}

The chromatin-associated human mobility group (HMG) superfamily plays crucial role in the regulation of chromatin structure, post-translational modifications, and rates of transcription, replication, and recombination. It is classified into three distinct families based on the structure of the DNAbinding domain and the substrate-binding specificity: HMGAT-hook (HMGA) family, HMG-box (HMGB) family, and HMG-nucleosome binding (HMGN) family [1]. 
A member of HMGB family HMGB1 has been shown to be released from monocytes, macrophages, neutrophils, and dendritic cells, and act as a key proinflammatory factor and late-acting distal inflammatory mediator during cell injury, necrosis and inflammation [2, 3]. In oral cavity, lipopolysaccharide (LPS) of two major periodontal pathogens, Aggregatibacter.actinomycetemcomitans and Porphyromonas. gingivalis, induced HMGB1 secretion from human gingival fibroblast (HGF) which might contribute to periodontal tissue destruction [3]. Compared with other proinflammatory cytokines such as Interleukin-1 $\beta($ IL-, 1 $\beta)$, IL-6, IL-8 and Tumor necrosis factor- $\alpha(\mathrm{TNF}-\alpha)$, the release of HMGB1 is retarded for several hours and it can be promoted by IL-1 $\beta$ and TNF- $\alpha$. Consequently, the released HMGB1 activates the monocytes to stimulate further release of proinflammation cytokines such as TNF- $\alpha$, IL-1 $\beta$ and IL-6, forming a positive feedback loop [4, $5]$.

Interestingly, our previous studies demonstrate that HMGN2, a member of HMGN family, acts as potentially important factors in the innate immunity of human reproductive tract $[6,7]$, and regulates LPS induced $\beta$-defensin expression in rat genital tract inflammation model [7, 8]. During periodontitis the affected gingival tissues and gingival crevicular fluid (GCF) are characterize by elevated levels of inflammation-associated Matrix metalloproteinase -25 (MMP$25)$ and -26 and human $\beta$-denfeisn-1 and -2 [9]. Therefore, we wonder whether the upregulation of these molecules is mediated by abnormal expression of HMGB1 and HMGN2 in periodontitis. In the current study, we compared the expression of HMGB1 and HMGN2 in gingival tissues and gingival crevicular fluid (GCF) in chronic periodontitis (CP), generalized aggressive periodontitis (G-AgP) patients and healthy subjects by real-time PCR. In addition, their expression in peri-implant crevicular fluid (PICF) in peri-implant crevicular fluid of peri-implantitis (PI-PICF) and normal patients was examined by Western blotting. Our results showed that HMGB1 and HMGN2 were increased in the gingival tissues of $\mathrm{CP}$ and G-AgP patients as well as in the GCF of $\mathrm{CP}$ and G-AgP patients and PICF in PI patients.

\section{MATERIAL AND METHODS}

\section{Patients}

The study population included 54 adults (18 females and 36 males, age 25-68 years). Among them, the patients in $\mathrm{CP}$ group ( $\mathrm{n}=15,4$ females and 11 males) were diagnosed with $\mathrm{CP}$ based on the following indices: visible plaque index, gingival bleeding, retentive calculus, pocket-probing depths and radiographic bone loss. The patients in $\mathrm{G}-\mathrm{AgP}$ group $(\mathrm{n}=14,4$ females and 10 males) were defined according to the diagnostic criteria as follows: assessment of pocket-probing depth, bleeding on probing, retentive calculus and visible plaque. The patients selected had deepened (>4 mm) pocket depths, bleeding on probing, but not much visible plaque or retentive calculus [9]. The patients in PI group $(n=15,7$ females and 8 males) were selected based on the inflammatory reaction with the loss of supporting bone in the tissues surrounding a function implant [19], The normal group $(n=10,3$ females and 7 males) comprised patients aged 18-57 years who were systemically healthy after peri-implant surgery. After the gingival tissue specimens and gingival crevicular fluid (GCF) samples were taken, the periodontal treatment was performed by a dentist at the Institute of Dentistry, West China College of Stomatology, Sichuan University.

All patients were healthy and had not used antibiotics during the previous 6 months. All participants were asked to give their consent in written form after the purpose and protocol of the study were instructed. This study was approved by Ethics Committee of State Key Laboratory of Oral Diseases of Sichuan University.

\section{Samples collection}

All inflamed gingival tissues specimens were collected from CP, G-Agp and PI patients before any periodontal treatment procedures as previously described [9]. The control 
specimens were collected during impacted third molar extraction surgery [10]. These samples were divided into: CP group, G-Apg group, PI group and control group.

PICF samples were collected from PI (PI-PICF) and normal group (Healthy-PICF), and GCF samples were collected from CP, G-Agp and control groups as previously described [9]. These samples were divided into: CP group, G-Apg group.

\section{RNA isolation and RT-PCR}

Total RNA was extracted from human gingival tissue specimens using Trizol (Invitrogen) following the manufacturer's instructions and the quality was examined by denaturing RNA electrophoresis in MOPS buffer. cDNA was synthesized from $1 \mu \mathrm{g}$ RNA using TOYOBO RT-PCR kit according to the manufacturer's instructions. Real-time PCR was performed usingSYBR green PCR kit (Bio-Rad) according to the manufactures' proocol. $5 \mu \mathrm{l}$ cDNA was amplified in a 25 $\mu l$ reaction mix containing $22.5 \mu$ of SYBR Green supermix and $1 \mu \mathrm{l}$ of each primer (Table 1 ). The amplification conditions were initial denaturation at $95{ }^{\circ} \mathrm{C}$ for $15 \mathrm{~min}$ followed by 35 cycles of denaturation at $95{ }^{\circ} \mathrm{C}$ for $15 \mathrm{sec}$, annealing at $58{ }^{\circ} \mathrm{C}$ for $15 \mathrm{sec}$ and elongation at $72{ }^{\circ} \mathrm{C}$ for $30 \mathrm{sec}$. To determine the level of HMGB1 and HMGN2 expression, the experiment was repeated three times under each experimental condition and the comparative threshold cycle method was employed with human $\beta$-actin as the internal control. Relative Expression $=2^{-}$ ${ }^{\Delta \Delta} \mathrm{CT}$.

Table 1. The primers used in real time PCR

\begin{tabular}{ll}
\hline primers & sequences \\
\hline HMGB1: ( 375bp) & 5'-CGC CAT GAG AAC TTC CTA CC -3'; \\
sense & 5'-CAC TTG GCC TTC CCT CTG TA -3' \\
antisense & 5'-CCAGCCATCAGCCATGAGGGT-3 \\
HMGN2: ( 245bp) & 5'-GGAGCCCTTTCTGAATCCGCA-3' \\
sense & \\
antisense & 5'-ATG TTT GAG ACC TTC AAC AC-3', \\
Huamn $\beta$-actin: ( 650bp) & 5'-CAC GTC ACA CTT CAT GAT GG-3' \\
sense &
\end{tabular}

\section{Western blotting}

The GCF and PICF samples were boiled in non-reducing Laemmli's buffer. The protein concentration of the extracts was determined using the BCA protein assay (Pierce) according to the manufacturer's instructions. The proteins were separated with SDS-PAGE and transferred to PVDF membranes. The membranes were incubated with HMGB1 (1:1000, Santa Cruz, USA), HMGN2 (1:500, Santa Cruz, USA) or $\beta$-actin (1:1000, Santa Cruz, USA) followed by incubation with the HRP conjugated anti-goat IgG antibody (Santa Cruz, USA). Finally the membranes were developed using ECL Plus reagent (Pierce, USA).

\section{ELISA}

IL-1 $\beta$, IL-6, IL-8, TNF- $\alpha$ and HMGB1 levels in GCF, PIPICF and healthy-PICF samples from different groups were determined using ELISA kits (R\&D, SAD) following the manufacturer's instructions.

\section{Data analysis}

Each experiment was performed from at least three dependent experiments, and each test condition was performed in duplicate. Values are shown as the Mean \pm SD (standard deviation) from multiple experiments as indicated. Statistical significance was determined using one-way analysis of variance (ANOVA) among the groups followed by the t-test. Differences were considered to be statistically significant at the level of $\mathrm{p}<0.05$.

\section{RESULTS}

Expression of HMGB1 and HMGN2 mRNA in control and diseased gingival tissues 
RT-PCR analysis showed that the expression of HMGB1 and HMGN2 mRNA was weak in gingival tissues in control group but was increased in $\mathrm{CP}$ and G-ApG groups. Furthermore, HMGB1 expression was the highest in CP group while HMGN2 expression did not show significant difference between $\mathrm{CP}$ and $\mathrm{G}-\mathrm{AgP}$ groups (Fig 1).

A

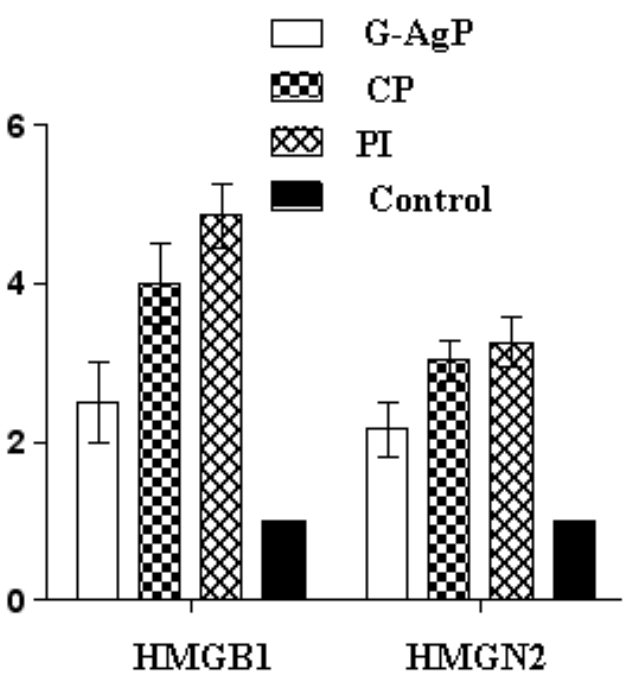

B

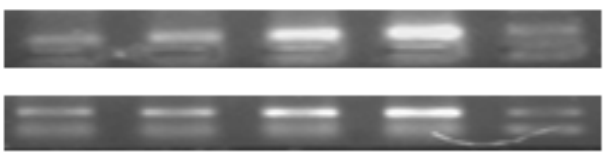

\section{HMGB1}

HMGN2

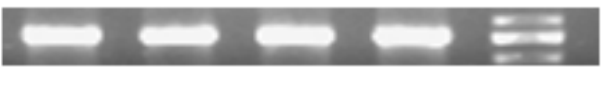

\section{$\beta$-actin}

\section{control G-ApG CP PI Maker}

Figure 1. The relative expression of HMGB1 and HMGN2 mRNA in gingival tissues from the patients in CP, G-AgP and healthy groups. A. Values were presented as mean \pm SD for at least three independent experiments performed in triplicate. The expressions of HMGB1 and HMGN2 in control group were normalized in 1.0. B. Representative RT-PCR images for HMGB1 and HMGN2 in four groups.

\section{HMGB1 and HMGN2 level in GCF and PICF}

Western blotting analysis demonstrated that while HMGB1 and HMGN2 proteins were weakly detected in the healthy GCF and PICF samples, their levels were much higher in GCF and PI-PICF samples from $\mathrm{CP}$ and G-AgP groups. The level of HMGB1 was higher in CP group than G-Agp group. In addition, the level of HMGN2 was less than that of HMGB1 in the same sample and showed no statistical difference between CP and G-Agp groups (Fig. 2).

\section{CP G-AgP PI-PICF Control Normal}

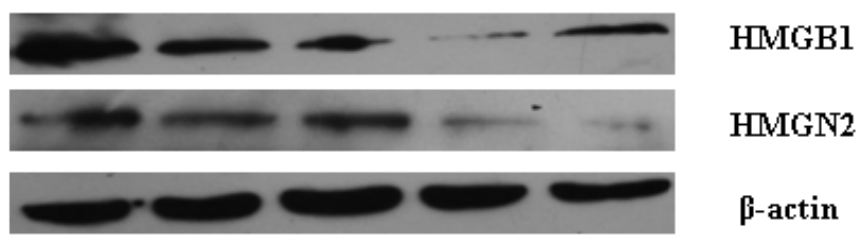

Figure 2. Representative Western blotting images for HMGB1 and HMGN2 expression in GCF from CP, G-AgP and control groups and in PICF in PI and normal group. $\beta$-actin served as the loading control.

\section{Concentration of cytokines in GCF and PICF}

To explore the potential link between cytokines and HMGB1 in GCF and PICF, we performed ELISA and the results showed that significantly higher concentrations of IL-1 $\beta$ and IL-6 (Table 2), IL-8 and TNF- $\alpha$ (Table 3), and HMGB1 (Table 4) were detected in GCF from G-AgP patients compared with that of the normal groups. Compare with GApg group, the concentrations of IL-1 $\beta$, IL-6, TNF- $\alpha$ and HMGB1 but not IL-8 were lower in CP group. Among all groups, the concentrations of IL-1 $\beta$, IL-6, IL- 8 and TNF- $\alpha$ were the highest in PI-PICF group. Among the cytokines, the concentration of IL-8 demonstrated the most significant change. The level of HMGB1 was the highest in the CP group. The concentrations of cytokines in normal groups were higher than those in healthy controls, but the differences were not statistically significant. 
Table 2. Concentration of proinflammatory cytokines IL-1, IL-6 in GCF of CP, G-AgP, Healthy and PICF in PI-PICF and normal group $(\mathrm{pg} / \mathrm{ml})$

\begin{tabular}{llllll}
\hline IL-1 $\beta$ & $\mathbf{X}$ & SD & med & SE & N \\
\hline CP & 645.2 & 228.9 & 543.0 & 52.7 & 15 \\
G-AgP & 708.9 & 323.5 & 613.5 & 123.8 & 15 \\
PI-PICF & 1421.4 & 1521.6 & 1121.5 & 526.3 & 15 \\
Healthy & 14.5 & 16.7 & 8.0 & 3.4 & 15 \\
Normal & 21.8 & 17.8 & 9.5 & 4.2 & 15 \\
IL-6 & $\mathrm{X}$ & SD & med & SE & N \\
CP & 498.5 & 129.6 & 289.0 & 66.8 & 15 \\
G-AgP & 1703.5 & 848.7 & 1456.0 & 724.6 & 15 \\
PI-PICF & 1567.9 & 772.3 & 1121.5 & 683.5 & 15 \\
Healthy & 24.9 & 25.7 & 11.0 & 10.8 & 15 \\
Normal & 35.4 & 17.1 & 20.5 & 13.6 & 15 \\
\hline X, mean; SD, standard deviation; med, median; SE, standard error. & & &
\end{tabular}

$\mathrm{X}$, mean; SD, standard deviation; med, median; SE, standard error.

Table 3. Concentration of proinflammatory cytokines (IL-8, TNF- $\alpha$ ) in GCF of CP, G-AgP, Healthy and PICF in PI-PICF and normal group $(\mathrm{pg} / \mathrm{ml})$

\begin{tabular}{llllll}
\hline IL-8 & X & SD & med & SE & N \\
\hline CP & 2450.3 & 875.6 & 1723.4 .0 & 753.2 & 15 \\
G-AgP & 1821.4 & 610.2 & 1559.0 & 438.2 & 15 \\
PI-PICF & 2719.8 & 258.4 & 1987.5 & 193.9 & 15 \\
Healthy & 68.5 & 23.8 & 32.0 & 11.9 & 15 \\
Normal & 79.1 & 29.8 & 43.5 & 17.3 & 15 \\
TNF- $\alpha$ & $\mathrm{X}$ & $\mathrm{SD}$ & med & SE & $\mathrm{N}$ \\
CP & 25.9 & 11.4 & 12.0 & 6.5 & 15 \\
G-AgP & 33.4 & 8.2 & 16.0 & 4.6 & 15 \\
PI-PICF & 36.2 & 19.5 & 21.5 & 12.2 & 15 \\
Healthy & 10.8 & 12.3 & 8.0 & 8.8 & 15 \\
Normal & 22.7 & 9.2 & 22.5 & 3.1 & 15 \\
\hline X, & & & & \\
\hline
\end{tabular}

$\mathrm{X}$, mean; SD, standard deviation; med, median; SE, standard error.

Table 4. Concentration of proinflammatory cytokines (HMGB1) in GCF of CP, G-AgP, Healthy and PICF in PI-PICF and normal group $(\mathrm{pg} / \mathrm{ml})$

\begin{tabular}{llllll}
\hline HMGB1 & X & SD & med & SE & N \\
\hline CP & 1892.4 & 919.3 & 1457.0 & 723.4 & 15 \\
G-AgP & 721.7 & 237.6 & 650.5 & 158.3 & 15 \\
PI-PICF & 877.9 & 121.3 & 785.5 & 98.4 & 15 \\
Healthy & 19.6 & 8.2 & 14.0 & 6.7 & 15 \\
Normal & 11.3 & 12.6 & 9.5 & 8.6 & 15 \\
\hline
\end{tabular}

X, mean; SD, standard deviation; med, median; SE, standard error. 


\section{DISSCUSSION}

In the present study, we investigated the expression of HMGB1 and HMGN2 mRNA and protein in gingival tissues and GCF from $\mathrm{CP}$ and $\mathrm{AgP}$ patients, and in PGCF from PI and healthy peri-implant patients.. In addition, by ELISA we detected the concentrations of proinflammatory factors in these groups.

Previous studies have provided evidence that HMGN2 is a novel antimicrobial effector against bacterial invasion [11]. In this report, we found that HMGN2 expression was increased in inflamed gingival tissues and GCF from $\mathrm{CP}$ and G-ApG groups, suggesting that the increased production and release of HMGN2 may be a anti-inflammatory response to protect the gingival tissues. In addition, HMGN2 expression was increased in PI-PICF which indicates that HMGN2 may also defend the peri-implant against the inflammation.

In contrast, HMGB1 is considered to play a proinflammatory function. For example, intratracheal injection of HMGB1 led to the acute lung injury and triggered the inflammation by necrosis cells [12]. Furthermore, anti-HMGB1 antibody was administrated to protect mice against LPSinduced lethality and decrease the severity of acute lung injury and the release of proinflammatory factor such as IL-1 $\beta$ and TNF- $\alpha[13,14]$. HMGB1 stimulated the release of IL-6 and IL11 from human periodontal ligament (PDL) cells via receptors including RAGE, TLR2 and TLR4 [15]. In addition, increased amount of extracellular HMGB1 was observed in salivary glands of patients with Sjögren's syndrome and HMBG1 together with TNF- $\alpha$ and IL- $1 \beta$ could form a proinflammatory loop to promote the chronic features of the glandular inflammation [16]. Consistent with these previous studies, we found that the expression of HMGB1 was highest in the gingival tissues and GCF from CP patients. More significantly, our results demonstrated that the high expression of HMGB1 was accompanied by increased concentrations of several cytokines, suggesting that HMGB1 and these cytokines form a positive feedback loop to promote inflammation in chronic periodontitis $[17,18]$. Among various cytokines, IL-1 $\beta$ promoted the release of HMGB1 from activated astrocytes through ERK/MAP kinase signaling and CRM1 signaling [19]. HMGB1 mRNA and protein were also produced by macrophages and cardiomyocytes followed by increased NFkappaB binding activity with consecutively sustained TNF- $\alpha$ and IL-6 expression [20]. HMGB1 activates HUVECs (human umbilic vein endothelial cells) to produce multiple inflammatory cytokines, such as IFN- $\gamma$, IL-6 and IL-8 [21].

It has been documented that the concentration of IL- $1 \beta$, IL-6 and IL-8 in GCF was significantly elevated in periodontitis [22]. In addition, IL-1 $\beta$, TNF- $\alpha$, MIP- $1 \alpha$ and IL-8 in PICF could be the prognostic markers of implant failure because their concentrations were significantly increased with advanced mucositis compared with the early stage [23]. Our results showed that there were statistically significant differences in the concentration of IL-1 $\beta$, IL- 6 and IL- 8 in GCF of CP, G-AgP, healthy and PICF in PI-PICF and normal group. These data suggest that these cytokines could stimulate HMGB1 production in later stage and then HMGB1 further promotes the release of these cytokines in periodontitis and peri-implant conditions.

In conclusion, to our knowledge, this is the first study reporting that the expression of HMGB1 and HMGN2 was increased in the gingival tissues and GCF in $\mathrm{CP}$ and G-AgP and the PICF in PICF. Our data suggest that HMGB1 may be a potential target for the therapy of periodontitis and PI.

\section{ACKONWLEDGMENTS}

This study was supported by grants from the National Natural Science Foundation of China (No. 30671963, No. 30470763, and CMB 98681).

\section{REFERENCE}

1. Abraham, E.; Arcaroli, J.; Carmody, A.; Wang, H.; Tracey, K.J. (2000). HMG-1 as a mediator of acute lung inflammation. J Immunol. 165: 2950-2954. 
2. Agnello, D.; Wang, H.; Yang, H.; Tracey, K.J.; Ghezzi, P. (2002). HMGB-1, a DNA-binding protein with cytokine activity, induces brain TNF and IL- 6 production, and mediates anorexia and taste aversion. Cytokine; 4: 231-236.

3. Andersson, U.; Wang, H.; Palmblad, K.; Aveberger, A.C.; Bloom, O.; Erlandsson-Harris, H.; Janson, A.; Kokkola, R.; Zhang, M.; Yang, H.; Tracey, K.J. (2000). High mobility group 1 protein (HMG-1) stimulates proinflammatory cytokine synthesis in human monocytes. J Exp Med. 192: $565-570$.

4. Bustin, M. (2001). Chromatin unfolding and activation by HMGN chromosomal proteins. Trends Biochem Sci. 26: 431-437.

5. Ek, M.; Popovic, K.; Harris, H.E.; Nauclér, C.S.; Wahren-Herlenius, M. (2006). Increased extracellular levels of the novel proinflammatory cytokine high mobility group box chromosomal protein 1 in minor salivary glands of patients with Sjögren's syndrome. Arthritis Rheum 54: 2289-94.

6. Feng, Y.; Huang, N.; Wu, Q.; Wang, B. (2005). HMGN2: a novel antimicrobial effector molecule of human mononuclear leukocytes? $J$ Leukoc Biol 78:1136-41.

7. Hasegawa, N. (2008). [Effect of high mobility group box 1 (HMGB1) in cultured human periodontal ligament cells] Kokubyo Gakkai Zasshi. 75: 155-61.

8. Hayakawa, K.; Arai, K.; Lo, E.H. (2010). Role of ERK map kinase and CRM1 in IL-1beta-stimulated release of HMGB1 from cortical astrocytes. Glia 58: 1007-15.

9. Jeon, E.J.; Kwak, H.W.; Song, J.H.; Lee, Y.W.; Chung, J.W.; Choi, J.C.; Shin, J.W.; Park, I.W.; Choi, B.W.; Kim, J.Y. (2007). Proinflammatory Effects of High Mobility Group B1 (HMGB1) Versus LPS and the Mechanism of IL-8 Promoter Stimulation by HMGB1. Tuberculosis and Respiratory Diseases 62: 299-307.

10. Kuula, H.; Salo, T.; Pirilä, E.; Hagström, J.; Luomanen, M.; GutierrezFernandez, A.; Romanos, G.E.; Sorsa, T. (2008). Human beta-defensin-1 and -2 and matrix metalloproteinase-25 and -26 expression in chronic and aggressive periodontitis and in peri-implantitis. Arch Oral Biol 53:175-86.

11. Kuula, H.; Salo, T.; Pirilä, E.; Hagström, J.; Luomanen, M.; GutierrezFernandez, A.; Romanos, G.E.; Sorsa, T. (2008). Human beta-defensin-1 and -2 and matrix m etalloproteinase- 25 and -26 expression in chronic and aggressive periodontitis and in peri-implantitis. Arch Oral Biol 53: 175-86.

12. Li, J.; Wang, H.; Mason, J.M.; Levine, J.; Yu, M.; Czura, C.J.; Tracey, K.J.; Yang, H. (2004). Recombinant HMGB1 with cytokine-stimulating activity. J. Immunol. Methods 289: 211-223.
13. Li, M.; Pan, X.L.; Wang, L.L.; Feng, Y.; Huang, N.; Wu, Q.; Li, X.; Wang, B.Y. (2005). [Study of antmicrobial mechanisms of human cervical mucus: isolation and characterization of antibacterial polypeptides]. Zhonghua Yi Xue Za Zhi. 85:1109-12.

14. Liu, J.H.; Li, Z.J.; Tang, J.; Liu, Y.W.; Zhao, L.; Deng, P.; Jiang, Y. (2006). [High mobility group box-1 protein activates endothelial cells to produce cytokines and has synergistic effect with lipopolysaccharide in inducing interleukin-6 release]. Zhonghua Yi Xue Za Zhi. 86:1191-5.

15. Mak, P.; Siwek, M.; Pohl, J. (2007). Adam Dubin menstrual hemocidin $\mathrm{HbB} 115-146$ is an cidophilic antibacterial peptide potentiating the activity of human efensins, cathelicidin and lysozyme. Am J Reprod Immunol 57: 81-91.

16. Ming, L.; Xiaoling, P.; Yan, L.; Lili, W.; Qi, W.; Xiyong, Y.; Boyao, W.; Ning, H. (2007). Purification of antimicrobial factors from human cervical mucus. Hum Reprod 22:1810-5.

17. Pavare, J.; Grope, I.; Kalnins, I.; Gardovska, D. (2010). High-mobility group box-1 protein, lipopolysaccharide-binding protein, interleukin-6 and $\mathrm{C}$-reactive protein in children with community acquired infections and bacteraemia: a prospective study. BMC Infect Dis 10: 28.

18. Petković, A.B.; Matić, S.M.; Stamatović, N.V.; Vojvodić, D.V.; Todorović, T.M.; Lazić, Z.R.; Kozomara, R.J. (2010). Proinflammatory cytokines (IL-1beta and TNF-alpha) and chemokines (IL-8 and MIP1alpha) as markers of peri-implant tissue condition. Int J Oral Maxillofac Surg 39:478-85.

19. Tervahartiala, T.; Pirila, E.; Ceponis, A. (2000). The in vivo expression of the collagenolytic matrix metalloproteinases (MMP-2, -8, -13, and 14) and matrilysin (MMP-7) in adult and localized juvenile periodontitis. J Dent Res 79: 1969-77.

20. Ueno, H.; Matsuda, T.; Hashimoto, S.; Amaya, F.; Kitamura, Y. (2004). Contributions of high mobility group box protein in experimental and clinical acute lung injury. Am J Respir Crit Care Med 170: 1310-1316.

21. Watanabe, T.; Kubota, S.; Nagaya, M.; Ozaki, S.; Nagafuchi, H.; Akashi, K.; Taira, Y.; Tsukikawa, S.; Oowada, S.; Nakano, S. (2005). The role of HMGB-1 on the development of necrosis during hepatic ischemia and hepatic ischemia/reperfusion injury in mice. J Surg Res 124: 59-66.

22. Yang, H.; Hreggvidsdottir, H.S.; Palmblad, K.; Wang, H.; Ochani, M.; Li, J.; Lu, B.; Chavan, S.; Rosas-Ballina, M.; Al-Abed, Y.; Akira, S.; Bierhaus, A.; Erlandsson-Harris, H.; Andersson, U.; Tracey, K.J. (2010). A critical cysteine is required for HMGB1 binding to Toll-like receptor 4 and activation of macrophage cytokine release. Proc Natl Acad Sci U S A 107:11942-7.

23. Yang, H.; Wang, H.; Czura, C.J.; Tracey, K.J. (2005). The cytokine activity of HMGB1. J. Leukoc. Biol. 23: 78:1-8. 